\title{
The Importance of Understanding Differences in a Critical Size Model: a Preliminary In Vivo Study Using Tibia and Parietal Bone to Evaluate the Reaction with Different Biomaterials
}

\author{
Guinea Brasil Camargo Cardoso ${ }^{a, b} * \mathbb{D}$, Erivelto Luis Chacon ${ }^{c}$, Luis Ricardo Barbosa Maiac, Cecília \\ Amélia de Carvalho Zavaglia ${ }^{a, b}$, Marcelo Rodrigues da Cunha \\ ${ }^{1}$ Departamento de Engenharia de Materiais, Faculdade de Engenharia Mecânica, Universidade \\ Estadual de Campinas, Campinas, SP, Brasil \\ ${ }^{2}$ Institutos Nacionais de Ciência e Tecnologia em Biofabricação - INCT Biofabris, Brasília, DF, Brasil \\ ${ }^{3}$ Departamento de Morfologia e Patologia, Faculdade de Medicina de Jundiaí, Jundiai, SP, Brasil
}

Received: July 11, 2018; Revised: September 18, 2018; Accepted: November 16, 2018

\begin{abstract}
Many researches aim to develop different biomaterials that are compatible with natural tissues. In vitro and in vivo tests are used to evaluate this potential. Our aim was to report the importance of the critical defect's location for in vivo assays, to evaluate this approach; in vivo studies were performed, using different compositions of biomaterials in two critical size defects: tibia and parietal bone. Polycaprolactone was used as the main polymeric matrix with and without addition of hydroxyapatite. In vivo studies on the standard critical size defect in tibia and parietal bone were performed using Wistars models: $3 \times 2$ and $5 \times 1$ dimensions, respectively. The animals were sacrificed after 32 days; neobone formation was assessed with the histological data. The in vivo data demonstrated differences between the tibia and parietal bone groups: the influence of the bone on the neobone's formation was notable. All the tibia defect samples had greater neobone volume when compared to the parietal data. Indeed, these bones have distinct embryology, influence of mechanical forces and vascularization rate that are well known; moreover, these characteristics were demonstrated to be critical for neobone formation.
\end{abstract}

Keywords: Defect model, Tibia bone, Cranial bone, Polycaprolactone, Hydroxyapatite.

\section{Introduction}

In vivo tests are procedures to evaluate the potential of different products and techniques to approve them for clinical use. Animal models are used to test the safety, efficacy and biocompatibility of the implant in relation to the body. Therefore, the correct selection is very important and needs to consider the animal's size, health, ability to stimulate conditions homologous or analogous to human conditions, cost, complexity of handling and availability. ${ }^{1}$

Pig, goat, rabbit, mouse/rat and dog are the main animal test models used to simulate a human in vivo environment and physical conditions for bone replacement. Each animal model/site has certain "critical-size defects", known as the smallest osseous defects that do not heal spontaneously over a long period of time (Table 1). ${ }^{2}$ Animal models are used to mimic the clinical situation as close as possible to the human aspect; however, each species has disadvantages compared with human conditions, e.g, denser trabecular network, shorter tibia and femur and high growth rates in pigs, whereas sheep have larger amount of bone ingrowth, and rabbit animal models have different bone anatomy, beside size and shape of bones with faster skeletal change and bone turnover.
Due to the handling complexity, cost and variation of each species, small animals (rodent) are often used as the ideal choice; however, significant physiological differences have been noted. ${ }^{1}$ Glucose concentration decreased in diabetic simulation rat models in the days following the implantation of a microdialysis probe, unlike humans for whom glucose levels increase and stabilize 3 days after implantation. ${ }^{3}$ In relation to bones, the small animals do not show Haversiantype remodeling in the cortex, and have limitations due to their small-sized long bones and thin and fragile cortices.

Table 1. Animals models and defect sites/sizes. All values are variable, due to the animals' age, gender, health and weight

\begin{tabular}{|c|c|c|}
\hline $\begin{array}{l}\text { Animal } \\
\text { model }\end{array}$ & Defect site & Defect size \\
\hline \multirow{3}{*}{ Rodent } & Calvaria & $\begin{array}{l}4 \mathrm{~mm} \text { diameter (nu mice) } 8 \mathrm{~mm} \\
\text { diameter (nude rat) }\end{array}$ \\
\hline & Distal femur & $\begin{array}{l}2 \mathrm{~mm} \text { in diameter with } 3 \mathrm{~mm} \text { in } \\
\text { length (Fisher male Wistar rat) }\end{array}$ \\
\hline & Midfemur & $5 \mathrm{~mm}$ in length (Nude rat) \\
\hline \multirow{3}{*}{ Rabbit } & Calvaria & $10 \mathrm{~mm}$ diameter per $1.2 \mathrm{~mm}$ \\
\hline & Femur & $\begin{array}{l}3 \mathrm{~mm} \text { in diameter with } 15 \mathrm{~mm} \\
\text { long }\end{array}$ \\
\hline & Ulna & $12 \mathrm{~mm}$ segment of midshaft ulnar \\
\hline \multirow{2}{*}{ Pig } & Craniofacial & $\begin{array}{l}10 \mathrm{~mm} \text { diameter with } 10 \mathrm{~mm} \text { depth } \\
\text { (porcine) }\end{array}$ \\
\hline & Tibial & $\begin{array}{l}11 \mathrm{~mm} \text { diameter with } 25 \mathrm{~mm} \\
\text { depth (Gottinger minipigs) }\end{array}$ \\
\hline
\end{tabular}


Even with these drawbacks, rat and mouse are the most commonly used animals for bone substitution tests.

The importance of the correct choice of animal model and site before testing in human beings is an indispensable issue. Different approaches are used in a wide number of animal test models, as well as different defect-critical sites. However, as the comparison between tibia and cranial bones has not been reported, our goal was to evaluate the investigation of cranial and tibia sites in Wistar models: an in-depth study was conducted comparing the two defectcritical sites used, indicating the difference of each anatomic site and its properties, which influence the result obtained.

\section{Material and Methods}

Poly ( $\varepsilon$-caprolactone) with $80 \mathrm{kDa}$ molar mass, used as the polymeric matrix, was supplied by Sigma-Aldrich. The sodium chloride $(\mathrm{NaCl})$, solvent (chloroform) and amphiphilic agent (oleic acid-OA) were purchased from Merck and Synth. The bioactive component was produced using $\alpha$-tricalcium phosphate as the primary reagent for the alkaline hydrolysis to obtain calcium-deficient hydroxyapatite whiskers (CDHA). ${ }^{4}$

To provide three-dimensional structures, the first step involved was to prepare a PCL/ $\mathrm{CHCl}_{3}$ solution in $12.5 \mathrm{w} / \mathrm{v} \%$ polymer/solvent concentration. $\mathrm{NaCl}$ particles were added to obtain $70 \mathrm{wt} \%$ porosity with diameters ranging from 177 to $350 \mu \mathrm{m}$. In the CDHA's composition, an amphiphilic agent was used to provide an efficient bond between the ceramic and polymer, in $0.5 \mathrm{wt} \%$ with $10 \%$ wt CDHA whiskers. The technique used to produce the scaffold was salt-leaching; after casting, it was processed for 48 hours for the solvent to evaporate, followed by 48 hours of salt-leaching in deionized water.

\subsection{Characterization}

Scanning Electron Microscopy (SEM) Jeol JXA 840 model was used to analyze the surface morphology of the scaffolds. The samples were stutter-coated using gold (Bal-tec SCD 050) with 40Ma,5 X $10^{7}$ conditions for 200 s.

To evaluate the Crystalline phase of the CDHA composition, X-Ray Diffraction (XRD was performed, using a DMAX 2200 X, Rigaku. The samples were run over a $2 \theta$ range between $20-40^{\circ}$ at a $0.05^{\circ} / \mathrm{s}$ scan speed. The XRD spectra were interpreted with JCPDS pattern 46-0905 to identify characteristic peaks of calcium-deficient hydroxyapatite $\left(26^{\circ}, 31.5^{\circ}\right.$ and $\left.33^{\circ}\right)$.

In vivo assay: Wistar rats approximately 6 to 7 months old were used under the approval of the Ethics Committee of the School of Medicine of Jundiaí (Protocol 276/13). Each group had 6 animals and was referenced in relation to biomaterial and site location. The control groups, without any biomaterial replacement, included tibia (T1) for the tibia's left site and parietal (P1) for the left parietal bone defect. A PCL scaffold - T2 and a PCL/CHDA scaffold were placed - T3 for tibia defect. The parietal bone defect groups were PCL and PCL/CDHA, P2 and P3, respectively. The animals were anesthetized with intramuscular injection of ketamine hydrochloride and xylazine hydrochloride at a 1:1 proportion $(0.10 \mathrm{mg} / 100 \mathrm{~g}$ body weight). After anesthesia and sterilization of the surgical site, different procedures were carried out for the tibia groups: a skin incision was made in the anterior region of the left limb to expose the anterior and proximal surface of the left tibia. A bone defect was drilled in the left tibia with a $3.0 \mathrm{~mm}$ trephine drill coupled to the pen of a mini-motor until the medullary canal was reached. ${ }^{5}$ For the parietal defect, the skull was shaved, and an incision was made in the skin to expose the parietal bones. The periosteum was detached and the $5 \mathrm{~mm}$ diameter defect was created using a trephine drill coupled to the pen of a mini-motor. Grafts $(3 \times 2$ or $5 \times 1 \mathrm{~mm}$, tibia and parietal respectively) were inserted in the defect followed by repositioning of the skin and musculature, and then sutured. The animals were sacrificed 32 days after the bone defect's creation with an overdose of the anesthetic, followed by $\mathrm{CO} 2$ inhalation. The left tibiae of groups 1 to 4 were removed; similarly, the skullcaps of the animals of groups 5 to 8 were also removed for morphological analysis of bone healing in the wound area. The samples were submitted to histological processing and semi-serial 5-mm cross-sections were cut and stained with hematoxylin-eosin and masson's trichrome. The bone formed at the implant site was quantified using the following formula: $\mathrm{Vv}=\mathrm{Pp} / \mathrm{Pt}(\%)$, where $\mathrm{Vv}$ is volume density or relative density; $\mathrm{Pp}$ is the number of points (line intersection) on the newly formed bone, and Pt is the total number of points of the system. ${ }^{5}$

For the statistical analyses, data was expressed as mean \pm SD. Comparisons between groups were made using ANOVA, followed by Tukey's correction factor for multiple comparisons as posthoc.

\section{Results}

Scaffolds are made to provide an adequate substrate for cell attachment and proliferation. To replace or repair bone tissue, these scaffolds need to have porosity, pores and composition similar or compatible with bone tissue. In our group, different compositions and techniques were developed to achieve the most appropriated substrate. ${ }^{6-9}$ PCL and PCL/ CDHA scaffolds were produced aiming at $70 \%$ porosity with 177 to $350 \mu \mathrm{m}$ pore diameter, using the casting and salt leaching method (Fig. 1A). Moreover, bioactive biomaterial was added in groups T3 and P3. CDHA whisker was used to provide osteoconduction capacity to the polymeric matrix, which means that the bone cells will be able to grow on this osteoconductive surface. This property results from the bone-like chemical composition of the ceramic that is similar to the ceramic part of the human bone's mineral 
called hydroxyapatite. Fig. 1B-C shows information about the calcium phosphate ceramic added: the CDHA crystals exhibited a needle-like morphology with a width ranging from 120 to $150 \mathrm{~nm}$ and length ranging from 2 to $4 \mu \mathrm{m}$; and crystalline peaks compatible with the international center for diffraction data (JCPDS) number 46-0905; the size and sharpness of the principal peaks $\left(26^{\circ}, 31.5\right.$ to $\left.33^{\circ}\right)$ indicate the presence of a crystalline ceramic without contamination.

After 32 days, the animals were sacrificed. The samples were observed to indicate the site of the critical defect; Fig. 2 shows images of these areas for T1 and P1, the control groups without addition of the biomaterial. By comparing these controls groups it is possible to note the defect in the parietal bone as opposed to the tibia samples for which the limitation is not clear. Corroborating the histological data, T1 features the formation of a new bone without organization of the structure; $\mathrm{P} 1$, however, did not feature any noticeable new bone formation.

Neo-bone formation was established in all groups; however, important differences were noted in each group. Fig. 3 shows the histological data of the biomaterial groups, as well as quantification of the neo-bone's volume. The new tissue had a different aspect in the comparison between tibia and parietal bone: a greater amount of new tissue and small islands were observed in the tibia groups; contrariwise, a concentrated neo tissue was observed on the extremity of the defect in the parietal samples. The bioactivity element - CDHA causes an induction for cell proliferation in both groups, indicated by the neo-bone islands located on all the tibia and parietal defects. Fig. 3C quantifies the neo-bone's volume in each group; statistical difference was observed in the tibia groups, a greater amount of neo-bone having been found in the samples with addition of CDHA (T3): $62.75 \pm 1.75$, followed by $\mathrm{T} 2$ and $\mathrm{T} 1$ with $48.58 \pm 0.32$ and $33.15 \pm 1.8$, respectively. The parietal defects had a smaller amount of new tissue, even with the osteogenic induction factor: $28.25 \pm 3.1,25.92 \pm 4.0$ and $14.45 \pm 1.0, \mathrm{P} 3, \mathrm{P} 2$ and $\mathrm{P} 1$ respectively.

\section{Discussion}

Multiple in vitro and in vivo assays were performed to assess the efficacy of the application of different biomaterials on bone tissue based on prior clinical tests. In vivo studies, besides providing information about the scaffold's composition and shape's potential, also contribute by supplying data about concentration and composition in relation to different induction factors. Additionally, an in vivo study with small animal models is recommended before advancing to larger animal models.
A
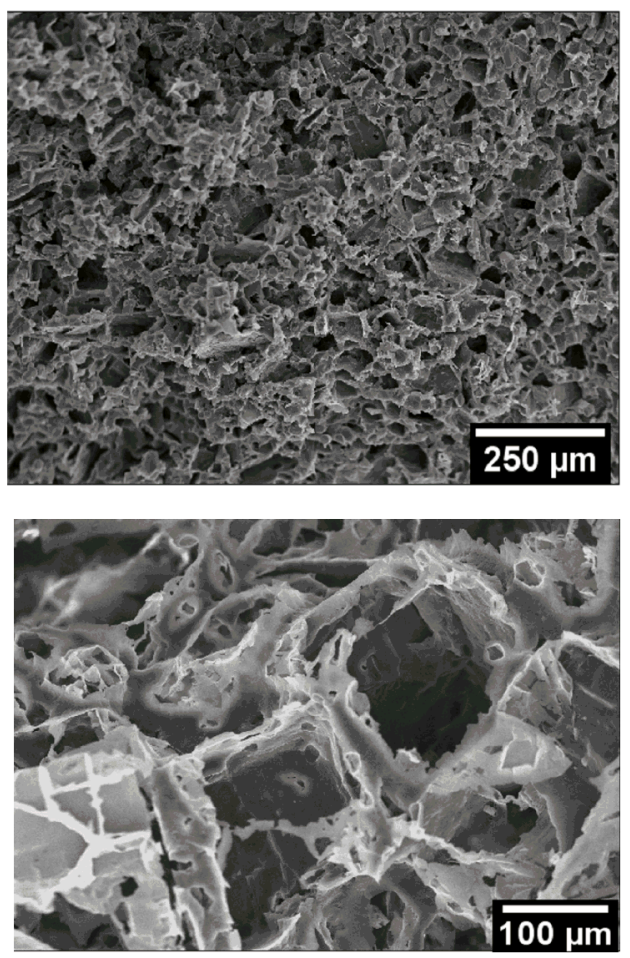

B
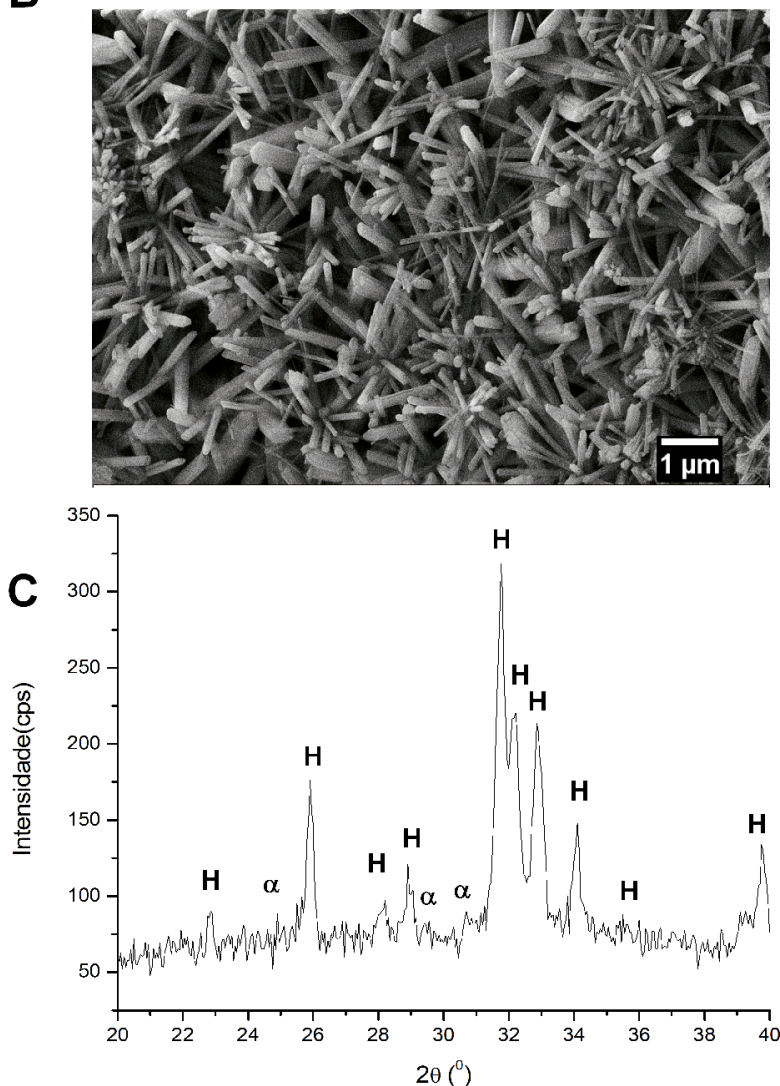

Figure 1. Scaffolds' characterization, A) SEM images of PCL scaffold (T2/P2), 250 and 100 $\mu \mathrm{m}$ scale bar; B) SEM image of CDHA whiskers added on T3 and P3, $1 \mu \mathrm{m}$ scale bar; C) CDHA XRD diffraction ( $\alpha$ and $\mathrm{H}$, indicate $\alpha$ - TCP and CDHA peaks, respectively). 


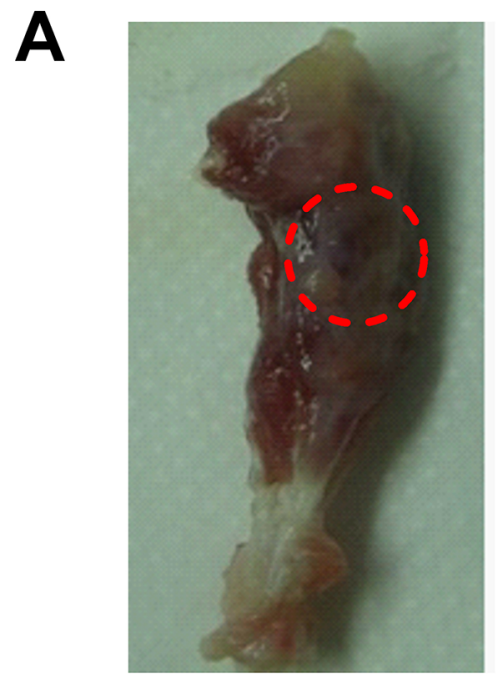

B
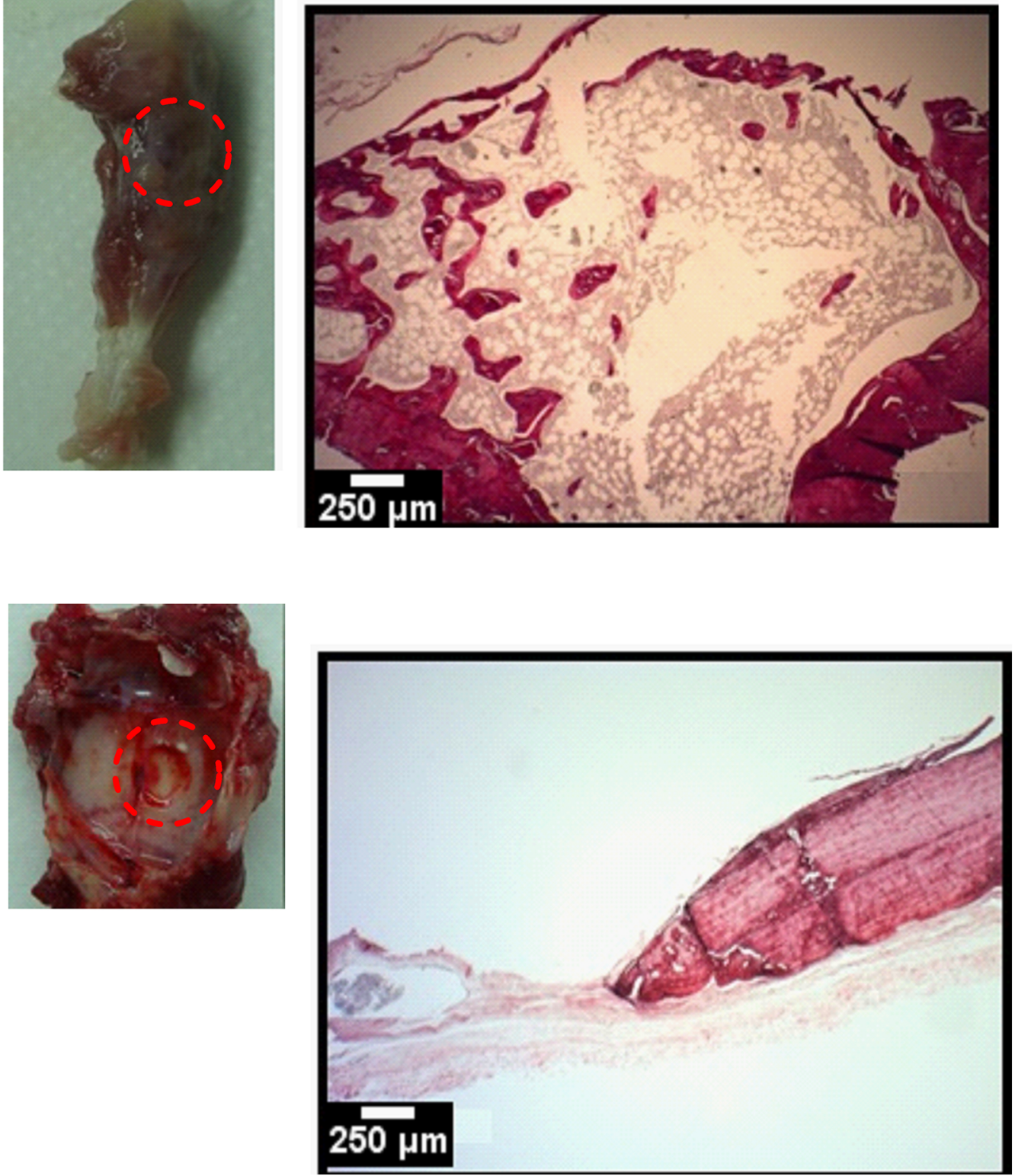

Figure 2. Control groups: A-B) Samples T1 and P1 after 32 days of culture with histological data, H\&E staining, 250 $\mu \mathrm{m}$ scale bar $250 \mu \mathrm{m}$; the red circle indicates the site of the critical defect.

Critical-size defect has been known since 1986, by Schmitz and Hollinger. ${ }^{10}$ Depending on animal and site this value can change, e.g., $8 \mathrm{~mm}$ in rats and $5 \mathrm{~mm}$ in mice for the calvaria bone. ${ }^{11}$

This research demonstrates different conclusions using PCL/CDHA and PCL biomaterials on both tibia and parietal bone sites. The PCL/CDHA biomaterial (T3-P3) had approximately double the control's neo-bone volume (T1-P1), and isolated neo-bone islands that indicate the potential induction factor of the bioceramic. As expected, without the bioactivity induction factor the data collected demonstrated smaller neo-bone volume: the PCL implanted in the parietal site (P2) exhibited $11 \%$ of neo-bone volume when compared to the control (P1), and 15\% when compared to the two tibia site groups (T2- T1). However, all the data collected from the tibia site provide expressive results, e.g, approximately $60 \%$ of neo-bone as opposed to $28 \%$ when implanted in the parietal site. Similar results were obtained by Lim et al., the authors suggested that this discrepancy was due to the influence of the remaining periosteum and mechanical load at the tibia defect site compared with the parietal bone site. ${ }^{12}$ Indeed, how can the same biomaterial 

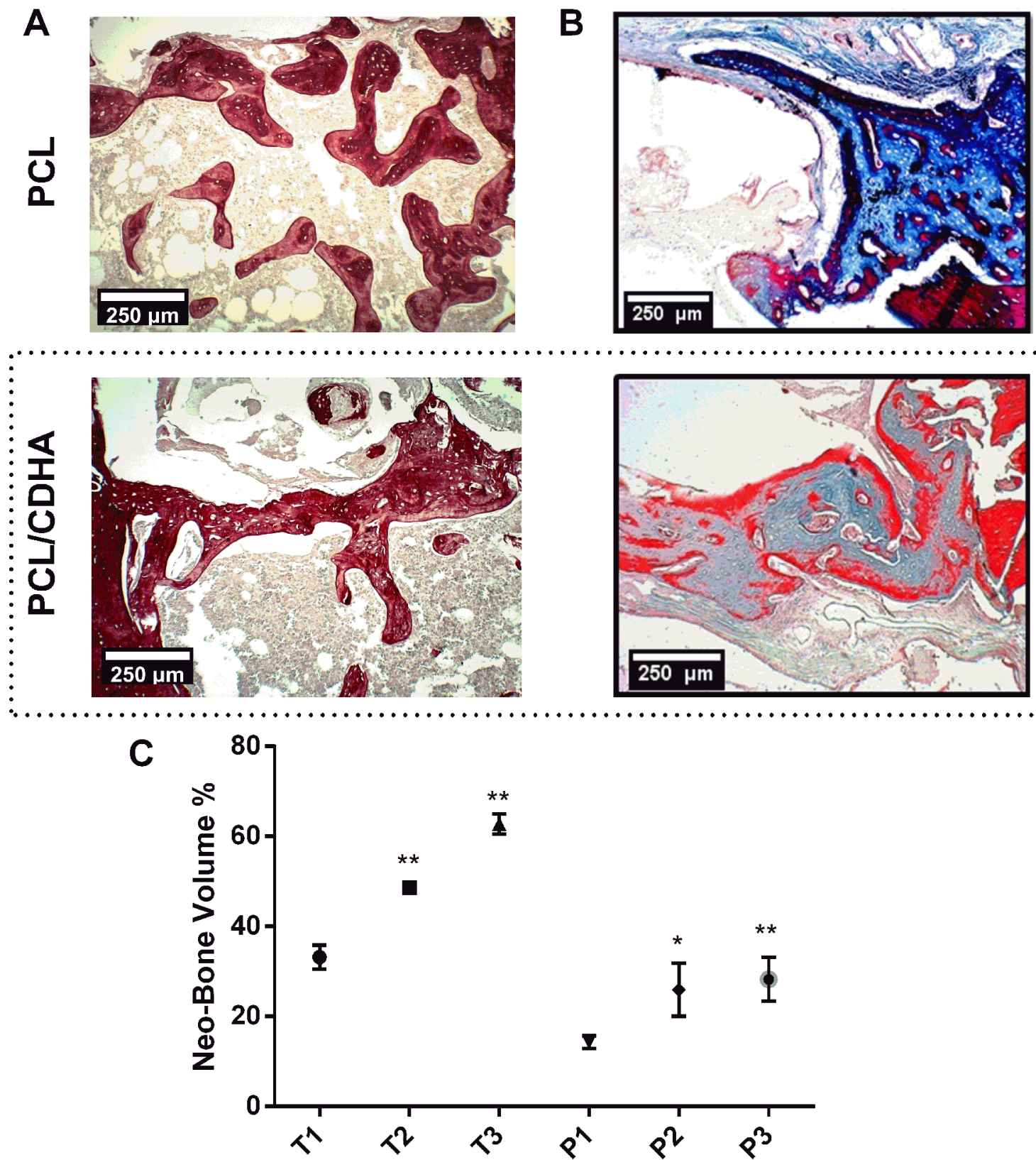

Figure 3. Histological data: A) Tibia groups with biomaterials PCL and PCL/CDHA, T2 and T3, respectively; B) Parietal groups, P2 and P3, $250 \mu \mathrm{m}$ scale bar; C) Quantification of neobone volume.*'s Indicates statistical differences between the control and the groups.

with identical composition and shape cause different results when implanted in tibia or parietal site models?

It is important to consider the differences between these two often-used models. Tibia is a long bone with greater vascular system; it is a useful model for reproducing a loading and unloading environment, also featuring endochondral ossification, as opposed to the calvaria bone, which features intramembranous ossification; the latter is known as the gold-standard model due to the fact its craniotomies are easy procedures with low morbidity rates. Also, since they are performed using a circular trephine, a large number of animals with reproducible results can be used.
Even though the calvaria bone is known as the goldstandard model, there are some important drawbacks. The first relates to the surgical procedures; they can damage or destroy the dura mater, which has a significant role in wound healing. The dura mater appears to be both the primary source of osteogenic cells and the source of osteoinductive factors, therefore its damaging may inhibit the healing process..$^{11} \mathrm{Also}$, it is well known that the mechanical load plays a decisive role in bone regeneration, and unfortunately it is not simulated when this model is adopted, which can induce an erroneous feedback of the biomaterial evaluated. ${ }^{13}$ 
Therefore, data of the tibia site demonstrate higher values due to its vascular system and mechanical load, as opposed to the parietal data, the latter having featured half the neo-bone volume due to the fact the main cellular source of this site is the dura mater, which has less vascularization when compared to the long bones' systems.

\section{Conclusion}

Animal models influence the response of bones to substitute biomaterials and thus the understanding of the biofunctionality, biocompatibility, osteo-conductivity and osteo-induction properties. Indeed, rats and goats have been proved to feature large differences of bone ingrowth speed and distance.${ }^{13}$ However, even larger animals are more suitable for mimicking human bone healing, the cost, variations between groups and surgery complexity being the main drawbacks. In this text, we have demonstrated the behavior of two different biomaterials: PCL and PCL/ CDHA (with the bioactivity induction factor) implanted in tibia and parietal sites using small animal models (Wistar rats). The conclusion based on our data was that the tibia site demonstrates greater neo-bone volume, due to the bone's anatomical characteristics: mechanical load and vascular system. Indeed, both site models are suitable for collecting data for further researches, but the formation rate should always be considered.

\section{Acknowledgements}

This work was funded by grants from the São Paulo Research Foundation (Fapesp 2009/54546-9 and 2013/19472$0)$. The authors thank Espaço da Escrita - Pró-Reitoria de Pesquisa - UNICAMP - for the language services provided.

Conflict of interest: The authors declare no conflict of interest.

\section{References}

1. Koschwanez HE, Reichert WM. In vitro, in vivo and post explantation testing of glucose-detecting biosensors: current methods and recommendations. Biomaterials. 2007;28(25):36873703.

2. Li Y, Chen SK, Li L, Qin L, Wang XL, Lai YX. Bone defect animal models for testing efficacy of bone substitute biomaterials. Journal of Orthopaedic Translation. 2015;3(3):95-104.
3. Wisniewski N, Rajamand N, Adamsson U, Lins PE, Reichert WM, Klitzman B, et al. Analyte flux through chronically implanted subcutaneous polyamide membranes differs in humans and rats. American Journal of Physiology, Endocrinology and Metabolism. 2002;282(6):E1316-1323.

4. Cardoso GBC, Ramos SLF, Rodas ACD, Higa OZ, Zavaglia CAC, Arruda ACF, et al. Scaffolds of poly (e-caprolactone) with whiskers of hydroxyapatite. Journal of Materials Science. 2010;45(18):4990-4993.

5. Da Cunha MR, Laraia IO, Franco GR, Miguel NN, Maciel AA, Dos Santos GR, et al. Osseointegration of Hydroxyapatite Implants in Rat Tibial Defects with Sciatic Nerve Injury. International Journal of Morphology. 2015;33(2):620-625.

6. Cardoso GB, Machado-Silva AB, Sabino M, Santos AR Jr, Zavaglia CA. Novel hybrid membrane of chitosan/poly (e-caprolactone) for tissue engineering. Biomatter. 2014;4. pii: e29508.

7. Cardoso CR, Favoreto S Jr, Oliveira LL, Vancim JO, Barban $\mathrm{GB}$, Ferraz DB, et al. Oleic acid modulation of the immune response in wound healing: A new approach for skin repair. Immunobiology. 2011;216(3):409-415.

8. Motisuke M, Gemma Mestres G, Renó CO, Carrodeguas RG, Zavaglia CAC, Ginebra MP. Influence of Si substitution on the reactivity of a-tricalcium phosphate. Materials Science and Engineering: C. 2017;75:816-821.

9. Larosa MA, Jardini AL, Zavaglia CAC, Kharmandayan P, Calderoni DR, Maciel Filho M. Microstructural and mechanical characterization of a custom-built implant manufactured in titanium alloy by direct metal laser sintering. Advances in Mechanical Engineering. 2014. DOI: $10.1155 / 2014 / 945819$

10. Schmitz JP, Hollinger JO. The critical size defect as an experimental model for craniomandibulofacial nonunions. Clinical Orthopaedics and Related Research. 1986;(205):299308 .

11. Cooper GM, Mooney MP, Gosain AK, Campbell PG, Losee JE, Huard J. Testing the critical-size in calvarial bone defects: revisiting the concept of a critical-size defect (CSD). Plastic Reconstructive Surgery. 2011;125(6):16851692.

12. McGovern JA, Griffin M, Hutmacher DW. Animal models for bone tissue engineering and modelling disease. Disease Models \& Mechanisms. 2018;11(4). pii: dmm033084.

13. Buma P, Schreurs W, Verdonschot N. Skeletal tissue engineering-from in vitro studies to large animal models. Biomaterials. 2004;25(9):1487-1495. 